\title{
A Doença Periodontal no Indivíduo com Fissura Labiopalatina
}

\author{
Periodontal Disease in the Individual with Cleft Lip and Palate
}

\author{
Luiz Evaristo Ricci Volpato; Ana Thereza de Saboia Campos Neves; ; Amanda Alves de Oliveira ${ }^{\mathrm{a}}$; Alexandre Meireles \\ Borba $^{\text {a }}$ Mateus Rodrigues Tonetto ${ }^{\text {; }}$ Cristhiane Almeida Leite da Silva ${ }^{\text {a }}$ Andreza Maria Fabio Aranha ${ }^{a}$
}

\begin{abstract}
aUniversidade de Cuiabá, Programa de Pós-Graduação Stricto Sensu em Ciências Odontológicas Integradas. MT, Brasil.
*E-mail: odontologiavolpato@uol.com.br
\end{abstract}

\begin{abstract}
Resumo
A doença periodontal abrange uma gama variada de condições inflamatórias da gengiva, osso e ligamento periodontal dependentes da microbiota do biofilme, de respostas imunes inatas, inflamatórias e adaptativas do hospedeiro e, ainda, fatores individuais genéticos e ambientais. Já as fissuras labiopalatinas são deformidades anatômicas decorrentes da fusão parcial dos processos maxilares nas primeiras semanas de vida intrauterina, especialmente, envolvendo o rebordo alveolar, resultando em alterações no arco dentário e vestíbulo, podendo provocar anomalias dentárias, mal posicionamento dentário e alterações na oclusão, como atresia maxilar e mordida cruzada. Este trabalho tem como objetivo revisar a literatura a respeito da prevalência de alterações periodontais em indivíduos com fissura labiopalatina em diferentes grupos etários e tipos de fissura, bem como comparar os achados com indivíduos sem fissura labiopalatina. Indivíduos com fissura labiopalatina têm sido considerados mais suscetíveis ao desenvolvimento de doença periodontal em função de sua condição anatômica particular e das consequências da fissura para o indivíduo, como muitas vezes o tratamento ortodôntico prolongado, que leva a maior acúmulo de biofilme. Entretanto, conhecimentos mais recentes têm demonstrado que a doença periodontal é uma alteração multifatorial mediada por complexos mecanismos, além do acúmulo de biofilme, como por exemplo, a condição imunológica individual, preponderante para a evolução da doença. Assim, novos estudos são necessários para compreender melhor o comportamento das doenças periodontais nos indivíduos com fissura labiopalatina.
\end{abstract}

Palavras-chaves: Fenda Labial. Fissura Palatina. Doenças Periodontais.

\begin{abstract}
Periodontal disease covers a wide range of inflammatory conditions of the gingiva, bone and periodontal ligament dependent on the biofilm microbiota, host's innate, inflammatory and adaptive immune responses, as well as individual genetic and environmental factors. Cleft lip and palate are anatomical deformities resulting from the maxillary processes partial fusion in the first weeks of intrauterine life, especially involving the alveolar ridge, resulting in changes in the dental arch and vestibule, which can also cause dental anomalies, dental malposition and changes in occlusion such as maxillary atresia and crossbite. This work aims to review the literature regarding the prevalence of periodontal changes in individuals with cleft lip and palate in different age groups and types of cleft, as well as to compare the findings with individuals without cleft lip and palate. Individuals with cleft lip and palate have been considered more susceptible to the development of periodontal disease due to their particular anatomical condition and the consequences of the cleft for the individual, such as often the prolonged orthodontic treatment that leads to greater biofilm accumulation. However, more recent knowledge has shown that periodontal disease is a multifactorial alteration mediated by complex mechanisms in addition to the biofilm accumulation, such as, for example, the individual immune condition, which is preponderant for the disease evolution. Thus, further studies are needed to better understand the periodontal diseases behavior in individuals with cleft lip and palate.
\end{abstract}

Keywords: Cleft Lip. Cleft Palate. Periodontal Diseases.

\section{Introdução}

Alterações no tecido periodontal de seres humanos não são achados recentes e sua prevalência passou a ser maior a partir da introdução da agricultura, nas sociedades neolíticas, segundo achados fósseis (ADLER et al., 2013). A doença periodontal abrange uma gama variada de condições inflamatórias crônicas da gengiva, osso e ligamento periodontal dependentes da microbiota do biofilme, de respostas imunes inatas, inflamatórias e adaptativas do hospedeiro e, ainda, fatores individuais genéticos e ambientais (EBERSOLE et al., 2013; SILVA et al., 2015; KINANE; STATHOPOULOU; PAPAPANOU, 2017).

As fissuras labiopalatinas (FLP) são deformidades anatômicas decorrentes da fusão parcial dos processos maxilares, nas primeiras semanas de vida intrauterina, especialmente, quando envolvem o rebordo alveolar, resultam em alterações no arco dentário e vestíbulo, podendo provocar outras anomalias dentárias, mal posicionamento dentário e alterações na oclusão, como atresia maxilar e mordida cruzada (SCHULTES; GAGGL; KARCHER, 1999; QUIRYNEN et al., 2003).

As alterações periodontais em pacientes com fissura labiopalatina têm sido estudadas, ao longo do tempo, utilizando diferentes metodologias, muitas vezes chegando a resultados conflitantes em relação a sua prevalência e severidade, quando analisados pacientes com diferentes tipos de fissura labioplalatina e quando esses são comparados com 
pacientes sem a deformidade congênita (PERDIKOGIANNI et al., 2009; GHELLER, 2018; TURNER et al., 1998, ALWAHADNI; ALHAIJA; AL-OMARI, 2005; PISEK et al., 2014; VEIGA et al., 2017; AHLUWALIA et al., 2004; BOLOOR; THOMAS, 2010; PAUL; BRANDT, 1998; HAZZA'A et al., 2011; CHOPRA et al., 2014; DE ALMEIDA et al., 2009; QUIRYNEN et al., 2003; MUTTHINENI; NUTALAPATI; KASAGANI, 2010; NAGAPPAN; JOHN, 2015; SALVI; BRAGGER; LANG, 2003; HUYNH-BA et al., 2009 ; NGUYEN et al., 2016; NEVES, 2020).

Assim, este trabalho tem como objetivo revisar a literatura a respeito da prevalência de alterações periodontais em indivíduos com fissura labiopalatina, em diferentes grupos etários e tipos de fissura, bem como comparar os achados com indivíduos sem fissura labiopalatina.

\section{Desenvolvimento}

\subsection{Doença periodontal}

Entende-se doença periodontal como uma variedade de condições inflamatórias crônicas da gengiva, osso e ligamento periodontal relacionadas à interação entre o biofilme dental e as respostas imunes inatas, inflamatórias e adaptativas desencadeadas. A natureza e a extensão da resposta são fundamentais na determinação da suscetibilidade e progressão da doença. Como consequência, há destruição dos tecidos que circundam e sustentam os dentes até a sua perda (EBERSOLE et al., 2013; SILVA et al., 2015; KINANE; STATHOPOULOU; PAPAPANOU, 2017).

A gengivite tem como característica ser uma forma reversível de doença periodontal, com a inflamação na margem gengival (KILIAN et al., 2016). No entanto, em indivíduos suscetíveis, a gengivite pode progredir para doença periodontal crônica, ou periodontite (KURGAN; KANTARCI, 2018). Na periodontite há destruição do periodonto, ou seja, das estruturas de suporte do dente, como a gengiva e o osso alveolar subjacente. O biofilme associado ao dente é necessário, mas não suficiente para induzir a periodontite, que depende da resposta inflamatória do hospedeiro a esse desafio microbiano (DARVEAU, 2010), além de outros fatores (NAGARAJAN et al., 2015; KINANE; STATHOPOULOU; PAPAPANOU, 2017).

Os tecidos periodontais são mantidos em um estado inflamatório "controlado" por meio do equilíbrio entre o sistema imunológico do hospedeiro e a microbiota, que compõe o biofilme dental. O desequilíbrio entre as espécies microbianas provoca uma disbiose da microbiota e, em um hospedeiro suscetível, pode levar ao desenvolvimento da periodontite (HAJISHENGALLIS, 2014).

Foram avanços recentes, como abordagens metagenômicas, que possibilitaram complementar a teoria de que apenas micro-organismos específicos estariam envolvidos no desenvolvimento da periodontite (SOCRANSKY et al., 1998; HAJISHENGALLIS; LAMONT, 2012). As bactérias estritamente anaeróbicas, conhecidas como patógenoschave, mesmo em quantidade muito pequena no biofilme, são capazes de causar disbiose na microbiota que leva à ruptura da homeostase com o hospedeiro (HAJISHENGALLIS; DARVEAU; CURTIS, 2012). Com isso, altera-se a quantidade e a organização dos micro-organismos comensais, que interagem fisicamente e metabolicamente com os patógenoschave. Entre os micro-organismos comensais há patógenos oportunistas, que podem induzir inflamação em hospedeiros suscetíveis (JIAO; HASEGAWA; INOHARA, 2014).

$\mathrm{O}$ estado inflamatório consequente oferece nutrientes, que por sua vez influenciam na composição da microbiota periodontal, favorecendo as espécies que se beneficiam dos produtos da degradação dos tecidos como aminoácidos em desfavor de espécies, que não são beneficiadas por essas alterações no microambiente, criando um ciclo de mais crescimento microbiano e mais inflamação (HAJISHENGALLIS; DARVEAU; CURTIS, 2012). Mudanças nas condições ambientais como concentração de oxigênio e pH também impulsionam a disbiose na comunidade microbiana (BARROS et al., 2016; KILIAN et al., 2016; VALM, 2019).

Assim, entende-se, atualmente, que o potencial danoso de micro-organismos como a Porphiromonasgingivalis está mais relacionado à manipulação da resposta do hospedeiro do que propriamente de sua capacidade de induzir inflamação (DARVEAU; HAJISHENGALLIS; CURTIS, 2012). Esta bactéria, mesmo em pouca abundância, atua corrompendo a sinalização do sistema imune inato, impactando o potencial de defesa, levando a alteração no crescimento e desenvolvimento da comunidade microbiana como um todo, mudando o relacionamento normalmente homeostático com o hospedeiro. Portanto, seu papel principal é muito mais orquestrar do que causar inflamação e perda óssea, que são amplamente mediadas pelos patobiontes sob condições de ruptura da homeostase (HAJISHENGALLIS et al., 2011; HAJISHENGALLIS; DARVEAU; CURTIS, 2012).

Entretanto, o colapso da homeostase tecidual não é necessariamente originário de dentro da comunidade microbiana. O equilíbrio microbiota-hospedeiro também pode ser rompido por imunodeficiências do hospedeiro, ou ainda, defeitos imunorregulatórios, doenças sistêmicas como diabetes, obesidade, fatores ambientais, como tabagismo, dieta e estresse, e modificações epigenéticas em resposta às mudanças ambientais. Esses fatores, de forma isolada ou em combinação, podem contribuir para o desequilíbrio homeostático. O envelhecimento é outro fator associado ao declínio da regulação e função imunológicas (HAJISHENGALLIS, 2014).

\subsection{Doença periodontal e fissura labiopalatina}

Diversos estudos apontaram que indivíduos com FLP apresentam um risco significativo à doença periodontal 
decorrente de particularidades inerentes à presença da fissura e de sua correção cirúrgica (SCHULTES; GAGGL; KARCHER, 1999; QUIRYNEN et al., 2003; AL-WAHADNI; ALHAIJA; AL-OMARI, 2005; PERDIKOGIANNI et al., 2009; HAZZA'A et al., 2011; NAGAPPAN; JOHN, 2015; NGUYEN et al., 2016).

Crianças e adolescentes com fissura labiopalatina (FLP) apresentam frequências de isolamento e proporções relativas de batérias aumentadas na microbiota subgengival dos dentes envolvidos na fissura (PERDIKOGIANNI et al., 2009).

Diferentes micro-organismos, como Aggregatibacter actinomycetemcomitans, Porphyromonas gingivalis, Tannerela forsythia e Streptococcus oralis foram detectadas e quantificadas em crianças e adolescentes com FLP. A quantidade foi estatisticamente superior no total de microorganismos presentes no biofilme subgengival dos indivíduos com FLP, quando comparado com os pacientes sem fissura (GHELLER, 2018). Uma possível razão para isso seria a pior higiene bucal percebida nesses indivíduos em comparação aos pacientes sem a deformidade congênita (TURNER et al., 1998, AL-WAHADNI; ALHAIJA; AL-OMARI, 2005; PISEK et al., 2014; VEIGA et al., 2017). A presença da fissura labiopalatina acarreta em maior retenção de alimentos nos locais dos defeitos, que também afeta a capacidade de manter uma boa higiene bucal (AHLUWALIA et al., 2004; BOLOOR; THOMAS, 2010).

Crianças e adolescentes com FLP, entre 6 e 16 anos, apresentaram higiene bucal ruim, maior retenção de alimentos e maior índice de placa bacteriana e gengivite, quando comparadas com crianças e adolescentes sem fissura (AHLUWALIA et al., 2004; VEIGA et al., 2017).

$\mathrm{O}$ acesso reduzido para higiene dos dentes ânterosuperiores, após o reparo cirúrgico da fissura labiopalatina, pode ser uma das explicações para condições desfavoráveis de higiene bucal nesses pacientes (PAUL; BRANDT, 1998). O tecido da mucosa é mais sensível do que o tecido aderido ao lado da fenda, o que explicaria esses indivíduos escovarem com muito menos profundidade, em que os dentes apresentam quantidades reduzidas de tecido aderido (MUTTHINENI; NUTALAPATI; KASAGANI, 2010). A presença da fissura labiopalatina resulta, ainda, em alterações oclusais, que também podem dificultar a higiene dos dentes (DAHLLÖF et al., 1989; PARADOWSKA-STOLARZ; KAWALA, 2014). Os índices de placa bacteriana e sangramento gengival são, significativamente, maiores em crianças com FLP em comparação com as crianças controle (AHLUWALIA et al., 2004; PERDIKOGIANNI et al., 2009; HAZZA'A et al., 2011; MUTTHINENI; NUTALAPATI; KASAGANI, 2010; PISEK et al., 2014; VEIGA et al., 2017).

Ao se comparar indivíduos com FLP, em dois grupos etários, de 10 a 15 anos e 16 a 28 anos com controles sem FLP, observou-se que o Índice de Placa Visível (IPV), Índice de Sangramento Gengival (ISG) e Profundidade de Sondagem (PS) apresentaram valores médios, significativamente, maiores naqueles com FLP do que nos controles, levando à conclusão, que aqueles apresentam risco aumentado de doença periodontal (AL-WAHADNI; ALHAIJA; AL-OMARI, 2005).

A higiene bucal e condição periodontal de 41 crianças e adolescentes com FLP foram analisadas e comparadas com 41 crianças controles, todos com faixa etária entre 4-18 anos. Os parâmetros clínicos avaliados foram: IPV, ISG e índice periodontal comunitário, e na área da FLP, os elementos dentários foram avaliados quanto ao ISG, PS e mobilidade dentária. Também foram feitas coletas de amostras de biofilme de 21 pacientes, selecionados de forma aleatória de cada grupo para análise microbiológica. Os indivíduos do grupo caso apresentaram IPV mais alto e pior higiene bucal em comparação ao grupo controle. Os dentes, na área da fenda, tinham PS e mobilidade dentária, significativamente, maiores em comparação com os dentes correspondentes no grupo controle. A análise microbiana não revelou diferenças significativas na composição da microbiota subgengival entre os grupos, porém os dentes próximos da região da FLP apresentaram bactérias periodontopatógenas de forma mais frequente (PERDIKOGIANNI et al., 2009).

Hazza'a et al. (2011) também encontraram acúmulo de placa e gengivite, significativamente, maiores em pacientes com FLP em comparação com seus controles, mostrando que indivíduos com fissura labiopalatina apresentam pior higiene bucal e demandam necessidade de medidas preventivas intensivas. Neste estudo, foram analisados 98 pacientes com FLP com idade entre 4-23 anos e comparados com 98 pacientes não afetados, pareados em sexo e idade. Para avaliação do estado periodontal, os índices IPV e ISG foram realizados.

Os mesmos índices, IPV e ISG foram utilizados por Chopra et al. (2014) para avaliar e comparar o estado de saúde gengival, em dois grupos compostos por crianças de 4-6 anos, sendo um grupo com 74 crianças com FLP e outro grupo controle com 48 crianças. Crianças com FLP apresentaram índice de placa e sangramento gengival mais altos do que as crianças do grupo controle.

Já Pisek et al. (2014) utilizaram os mesmos índices, porém analisando 68 crianças com FLP e 118 crianças sem FLP com idade entre 10 e 14 anos. As crianças com FLP apresentaram valores maiores para os índices em relação às crianças do grupo controle. Concluiu-se que crianças com FLP apresentam higiene bucal deficiente e alto índice de sangramento gengival, resultando em níveis elevados de gengivite.

Para a análise periodontal de 156 crianças de 5 a 18 anos, com FLP $(\mathrm{N}=78)$ e sem FLP $(\mathrm{N}=78)$, foram utilizados os índices IPV, ISG, PS e NIC, alem do diagnóstico de gengivite (localizada e generalizada) e periodontite (leve, moderada e severa). Os valores foram maiores nos indivíduos com FLP em relação ao grupo controle. Entre os pacientes com FLP, 49\% apresentaram saúde gengival, $22 \%$ apresentaram gengivite localizada e $29 \%$ apresentaram gengivite generalizada. No grupo controle, $75 \%$ apresentaram saúde gengival, 24\% gengivite localizada e $1 \%$ gengivite generalizada. Veiga et al. 
(2017) concluíram que a FLP é um fator determinante de risco para o desenvolvimento de inflamação gengival.

Com uma população na mesma faixa etária, sendo 60 indivíduos com FLP e 58 indivíduos controle, foi encontrada a presença de fissura labiopalatina que surge como determinante para o agravamento da gengivite. Foi observado também que $48 \%$ dos indivíduos com FLP apresentaram periodontite leve contra $22 \%$ do grupo controle. A microbiota do biofilme supragengival e subgengival apresentou quantidade total de micro-organismos superior nos indivíduos com FLP (GHELLER, 2018).

Para responder se os dentes próximos à fissura labiopalatina apresentam maior prevalência e gravidade de doença periodontal do que dentes em outros sítios, em um estudo transversal, foram analisados 400 indivíduos com FLP completa unilateral ou bilateral, com idade entre 15 e 49 anos, sem qualquer tratamento periodontal prévio. Foram avaliados os parâmetros clínicos de IPV, ISG, PS e Nível de Inserção Clínica (NIC) em todos os dentes, excluindo os terceiros molares. Entre os sextantes, 86,75\% apresentaram médias de PS menor ou igual a $3 \mathrm{~mm}$ e nenhum sextante exibiu média maior ou igual a $6 \mathrm{~mm}$. O sextante com FLP não apresentou maiores médias de IPV, ISG, PS e NIC. Houve sangramento gengival em $99,08 \%$ da amostra e presença de placa em $97,40 \%$. $\mathrm{O}$ tipo de fenda e gênero não foram fatores importantes para influenciar a prevalência da doença periodontal, porém a idade interferiu tanto na prevalência quanto na gravidade da doença. Os dentes na área da fissura não exibiram maiores índices de placa e índices de sangramento gengival comparados com as outras áreas. Ainda, segundo o estudo, muitas investigações em indivíduos com fissura avaliam apenas a área da fissura não levando em consideração se o indivíduo pertence a um grupo de risco ou se há perda de inserção periodontal em locais fora da área da fissura, em desacordo com os novos conceitos epidemiológicos da doença periodontal (DE ALMEIDA et al., 2009).

Já outro estudo avaliou os parâmetros periodontais e microbiológicos de 75 pacientes entre 8 e 20 anos com FLP unilateral completa, comparando dentes adjacentes à fissura com seus contralaterais. Os dentes próximos à fissura apresentaram aumento na profundidade de sondagem, no nível de inserção clínica e no índice de placa, quando comparados aos dentes contralaterais. Os exames microbiológicos não apresentaram diferença entre os diferentes sítios (QUIRYNEN et al., 2003).

Sessenta pacientes com fissuras foram divididos em três grupos, sendo FLP, fissura labial (FL) e fissura palatina isolada (FP) para análise do perfil periodontal. A higiene bucal foi avaliada pelo índice bucal simplificado e a saúde periodontal, pelo índice periodontal comunitário. A formação de cálculo dentário foi quase igualmente distribuída entre os sextantes posterior e anterior. Na maioria dos indivíduos, maior PS foi observada nos dentes adjacentes à fenda, no sextante anterior superior, e os dentes nas proximidades da fissura labiopalatina foram responsáveis, em $90 \%$ dos casos, pela alta incidência de sangramento no sextante anterior. A higiene bucal foi considerada ruim para todos os indivíduos. A presença de gengivite foi mais predominante em pacientes com FL e naqueles com FP e a periodontite foi mais presente em pacientes com FLP, com maior destruição periodontal nos dentes adjacentes à fissura labiopalatina (BOLOOR; THOMAS, 2010).

A higiene bucal e o perfil periodontal de pacientes com diferentes tipos de fissura também foi analisado e comparado por Mutthineni, Nutalapati e Kasagani (2010). A população do estudo foi composta por 60 pacientes com FP e 60 pacientes com fissura de lábio e palato completa unilateral (FLP) e foram registrados parâmetros de IPV, ISG, PS, NI, mobilidade dentária e quantidade de perda óssea radiográfica. A destruição periodontal foi maior no grupo de pacientes com FLP em comparação ao grupo com FP. A higiene bucal foi deficiente e o estado periodontal, indicado pelos índices PS, ISG, NIC, mobilidade dentária e quantidade radiográfica de perda óssea, foi pior nos pacientes com FLP.

Nagappan e John (2015), ao compararem o perfil periodontal de 80 pacientes com diferentes tipos de fissuras (FL, FP e FLP), em um estudo transversal, encontraram que entre os pacientes com FL, 15,4\% apresentaram sangramento à sondagem e 46,1\% acúmulo de cálculo. Dos pacientes com FP, 23\% apresentaram sangramento à sondagem e 27\% acúmulo de cálculo. E dos pacientes com FLP, 17,8\% apresentavam sangramento à sondagem e 11 39,2\% acúmulo de cálculo. O número médio de sextantes codificados para saudáveis e sangramento foi máximo entre os indivíduos com FP e o número médio de sextantes codificados para cálculo foi máximo entre os indivíduos com FLP.

Em estudo de coorte, ao longo de 14 anos, foi observado que tanto pacientes com FLP quanto com FL e FP apresentam elevado risco de progressão de doença periodontal, quando comparados aos indivíduos controle de idade similar. Encontrou-se grande acúmulo de biofilme resultando em reação inflamatória periodontal e perda significativa de inserção clínica neste estudo (SALVI; BRAGGER; LANG, 2003). A mesma coorte avaliada ao longo de 25 anos confirmou o maior risco de progressão da doença periodontal em indivíduos com fissura labiopalatina, independentemente do tipo (HUYNH-BA et al., 2009).

Nguyen et al. (2016) examinaram 78 pacientes com FLP com idade variando entre 1 e 54 anos, com média de 6 anos e a porcentagem de pacientes com bolsa periodontal entre 3,5 e $5,5 \mathrm{~mm}$ foi de $5,3 \%$.

Neves (2020) investigou relação da microbiota subgengival, do estresse oxidativo salivar e das citocinas inflamatórias presentes no fluido gengival crevicular (FGC), com doença periodontal em 41 pacientes com fissura labiopalatina com idade entre 15 e 44 anos e comparou com 40 indivíduos controle da mesma faixa etária. Os índices PS e NI foram, significativamente, superiores nos 
indivíduos com fissura jovens (15-19 anos) em comparação ao grupo controle, e a periodontite foi mais prevalente, tanto no grupo caso jovem quanto adulto (35-44 anos), em comparação aos grupos controle. Os níveis de IL-6 no FGC se mostraram estatisticamente mais elevados no grupo caso jovem em relação ao grupo controle. Foi observada correlação positiva entre os parâmetros clínicos e o estresse oxidativo e a capacidade antioxidante total nos indivíduos jovens com fissura. Tipo de fissura e uso de aparelho ortodôntico não interferiram na ocorrência de gengivite e periodontite. A presença de fissura aumentou a ocorrência de periodontite nos indivíduos jovens e adultos e, influenciou os níveis de IL-6 no FGC nos indivíduos jovens, porém não influenciou a microbiota subgengival, o estresse oxidativo e capacidade antioxidante total salivares e os níveis de IL-1 $\beta$ e MIP- $1 \alpha$ no FGC.

A grande variabilidade dos resultados encontrados em trabalhos, que analisaram a condição periodontal de pacientes com diferentes tipos de fissuras labiopalatinas pode ser explicada pela diferença nas metodologias e nas faixas etárias estudadas (HAZZA'A et al., 2011; PERDIKOGIANNI et al., 2009; VEIGA et al., 2017; GHELLER, 2018). Apesar disso, ressalta-se a importância do diagnóstico e tratamento precoce da doença periodontal nesses pacientes, especialmente, antes dos 30 anos (LANG; SCHATZLE; LOE, 2009; RAMSEIER et al., 2017).

\section{Conclusão}

Indivíduos com fissura labiopalatina têm sido considerados mais suscetíveis ao desenvolvimento de doença periodontal em função de sua condição anatômica particular e das conseqüências da fissura para o indivíduo, como muitas vezes o tratamento ortodôntico prolongado, que leva a maior acúmulo de biofilme. Entretanto, conhecimentos mais recentes têm demonstrado que a doença periodontal é uma alteração multifatorial mediada por complexos mecanismos, além do acúmulo de biofilme, como por exemplo, a condição imunológica individual preponderante para a evolução da doença. Assim, novos estudos são necessários para compreender melhor o comportamento das doenças periodontais nos indivíduos com fissura labiopalatina.

\section{Referências}

ADLER, C. et al. Sequencing ancient calcified dental plaque shows changes in oral microbiota with dietary shifts of the Neolithic and Industrial revolutions. Nat. Genet., v.45, n.4, p.450455, 2013. doi: 10.1038/ng.2536.

AHLUWALIA, M. et al. Dental caries, oral hygiene, and oral clearance in children with craniofacial disorders. J. Dent. Res., v.83, p.175-179, 2004. doi: 10.1177/154405910408300218.

AL-WAHADNI, A.; ALHAIJA, E. A.; AL-OMARI, M.A. Oral disease status of a sample of Jordanian people ages 10 to 28 with cleft lip and palate. Cleft Palate Craniofac J., v.42, n.3, p.304308, 2005. doi: 10.1597/03-161.1.

BARROS, S.P. et al. Gingival crevicular fluid as a source of biomarkers for periodontitis. Periodontol. 2000, v.7, n.1, p.5364, 2016. doi: 10.1111/prd.12107.

BOLOOR, V.; THOMAS, B. Comparison of periodontal status among patients with cleft lip, cleft palate, and cleft lip along with a cleft in palate and alveolus. J. Indian Soc. Periodontol., v.14, n.3, p.168-172, 2010. doi: 10.4103/0972-124X.75911.

CHOPRA, A.; LAKHANPAL, M.; RAO, N.C. et al. Oral health in 4-6 years children with cleft lip/palate: a case control study. $N$. Am. J. Med. Sci., v.6, n.6, p.266-269, 2014. doi: 10.4103/19472714.134371.

DAHLLÖF, G.; USSISOO-JOANDI, R.; IDEBERG, M. et al. Caries, gingivitis, and dental abnormalities in preschool children with cleft lip and/or palate. Cleft Palate J., v.26, n.3, p.233-238, 1989.

DARVEAU, R.P.; HAJISHENGALLIS, G.; CURTIS, M.A. Porphyromonasgingivalis as a potential community activist for disease. J. Dent. Res., v.91, n.9, p.816-820, 2012. doi: $10.1177 / 0022034512453589$.

DARVEAU, R. P. Periodontitis: a polymicrobial disruption of host homeostasis. Nat. Rev. Microbiol., v.8, n.7, p.481-490, 2010. doi: $10.1038 /$ nrmicro2337.

DE ALMEIDA, A.L.; GONZALEZ, M.K.; GREGHI, S.L. et al. Are teeth close to the cleft more susceptible to periodontal disease? Cleft Palate Craniofac J., v.46, n.2, p.161-165, 2009. doi: 10.1597/07-226.1.

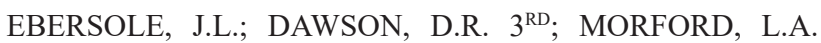
et al. Periodontal disease immunology: 'double indemnity' in protecting the host. Periodontol. 2000, v.62, n.1, p.163-202, 2013. doi: 10.1111/prd.12005.

GHELLER, S.A.P. Avaliação clínica e microbiológica do perfil periodontal de crianças e adolescentes com fissura labiopalatina: estudo de caso-controle. Cuiabá: Universidade de Cuiabá, 2018.

HAJISHENGALLIS, G.; LIANG, S.; PAYNE, M.A. et al. Lowabundance biofilm species orchestrates inflammatory periodontal disease through the commensal microbiota and complement. Cell. Host Microbe, v.10, n.5, p.497-506, 2011. doi: 10.1016/j. chom.2011.10.006.

HAJISHENGALLIS, G.; DARVEAU, R.P.; CURTIS, M.A. The keystone pathogen hypothesis. Nat. Rev. Microbiol., v.10, n.10, p.717-725, 2012. doi: 10.1038/nrmicro2873.

HAJISHENGALLIS, G. Immunomicrobial pathogenesis of periodontitis: keystones, pathobionts, and host response. Trends Immunol., v.35, n.1, p.3-11, 2014. doi: 10.1016/j.it.2013.09.001.

HAJISHENGALLIS, G.; LAMONT, R.J. Beyond the red complex and into more complexity: the polymicrobial synergy and dysbiosis (PSD) model of periodontal disease etiology. Mol Oral Microbiol., v.27, n.6, p.409-419, 2012. doi: 10.1111/j.20411014.2012.00663.x.

HAZZA'A, A.M.; RAWASHDEH, M.A.; AL-NIMRI, K. et al. Dental and oral hygiene status in Jordanian children with cleft lip and palate: a comparison between unilateral and bilateral clefts. Int. J. Dent. Hyg. v.9, n.1, p.30-36, 2011. doi: 10.1111/j.16015037.2009.00426.x.

HUYNH-BA, G.; BRÄGGER, U.; ZWAHLEN, M. Periodontal disease progression in subjects with orofacial clefts over a 25 year follow-up period. J. Clin. Periodontol. v.36, n.10, p.836842, 2009. doi: 10.1111/j.1600-051X.2009.01469.x.

JIAO, Y.; HASEGAWA, M.; INOHARA, N. The role of oral pathobionts in dysbiosis during periodontitis development. J. Dent. Res., v.93, n.6, p.539-546, 2014. doi: $10.1177 / 0022034514528212$. 
KILIAN, M.; CHAPPLE, I.L.; HANNIG, M. The oral microbiome - an update for oral healthcare professionals. Bras. Dent. J., v.221, n.10, p.657-666, 2016. doi: 10.1038/sj.bdj.2016.865.

KINANE, D.F.; STATHOPOULOU, P.G.; PAPAPANOU, P.N. Periodontal diseases. Nat. Rev Dis. Primers., v.3, n.17038, p.114, 2017. doi: 10.1038/nrdp.2017.38.

KURGAN, S.; KANTARCI, A. Molecular basis for immunohistochemical and inflammatory changes during progression of gingivitis to periodontitis. Periodontol. 2000, v.76, n.1, p.51-67, 2018. doi: 10.1111/prd.12146.

LANG, N.P.; SCHÄTZLE, M.A.; LÖE, H. Gingivitis as a risk factor in periodontal disease. J Clin. Periodontol., v. 36, p.3-8, 2009. doi: 10.1111/j.1600-051X.2009.01415.x.

MUTTHINENI, R.B.; NUTALAPATI, R.; KASAGANI, S.K. Comparison of oral hygiene and periodontal status in patients with clefts of palate and patients with unilateral cleft lip, palate and alveolus. J. Indian. Soc. Periodontol., v.14, n.4, p.236-240, 2010. doi: 10.4103/0972-124X.76928.

NAGAPPAN, N.; JOHN, J. Periodontal status among patients with Cleft Lip (CL), Cleft Palate (CP) and Cleft Lip, Alveolus and Palate (CLAP) in Chennai, India. A comparative study. J. Clin. Diagn. Res., v.9, n.3, p.53-55, 2015. doi: 10.7860/ JCDR/2015/11208.5723.

NAGARAJAN, R.. Patient-specific variations in biomarkers across gingivitis and periodontitis. PLoS One, v.25, n.9, p.1-15, 2015. doi: 10.1371/journal.pone.0136792.

NEVES, A.T.S.C. Análise do estresse oxidativo salivar, citocinas inflamatórias e microbiota subgengival e sua relação com doença periodontal em indivíduos com Fissura Labiopalatina. Tese (Doutorado em Ciências Odontológicas Integradas) Universidade de Cuiabá, Cuiabá, 2020.

NGUYEN, T.T.; NGO, L.Q.; PROMSUDTHI, A. et al. Salivary oxidative stress biomarkers in chronic periodontitis and acute coronary syndrome. Clin Oral Investig. v.21, n.7, p. 2345-2353, 2016. doi: 10.1007/s00784-016-2029-3.

PARADOWSKA-STOLARZ, A.; KAWALA, B. Occlusal disorders among patients with total clefts of lip, alveolar bone, and palate. Biomed Res. Int., v.2014, 583416. 2017. doi: $10.1155 / 2014 / 583416$.

PAUL, T.; BRANDT, R.S. Oral and dental health status of children with cleft lip and/or palate. Cleft Palate Craniofac J, v.35, n.4, p.329-332, 1998. doi: 10.1597/1545-1569_1998_035_0329_ oadhso 2.3.co 2 .

PERDIKOGIANNI, H. et al. Periodontal and microbiological parameters in children and adolescents with cleft lip and /or palate. Int. J. Paediatr. Dent., v.19, n.6, p.455-467, 2009. doi: 10.1111/j.1365-263X.2009.01020.x.

PISEK, A. et al. Oral health status and oral impacts on quality of life in early adolescent cleft patients. J. Med. Assoc. Thai., v. 97, n. 10, p. S7-S16, 2014.

QUIRYNEN, M. et al. A split-mouth study on periodontal and microbial parameters in children with complete unilateral cleft lip and palate. J. Clin. Periodontol., v.30, n.1, p.49-56, 2003. doi: 10.1034/j.1600-051x.2003.300108.x.

RAMSEIER, C.A. et al. Natural history of periodontitis: Disease progression and tooth loss over 40 years. J. Clin. Periodontol., v.44, n.12, p.1182-1191, 2017. doi: 10.1111/jcpe.12782.

SALVI, G.E.; BRAGGER, U.; LANG, N.P. Periodontal Attachment Loss Over 14 Years in Cleft Lip, Alveolus and Palate (CLAP, CL, CP) Subjects not Enrolled in a Supportive Periodontal Therapy Program. J. Clin. Periodontol., v.30, n.9, p.840-845, 2003. doi: 10.1034/j.1600-051x.2003.00390.x.

SCHUlTES, G.; GAGGL, A.; KARCHER, H. Comparison of periodontal disease in patients with clefts of palate and patients with unilateral clefts of lip, palate, and alveolus. Cleft Palate Craniofac J., v.36, n.4, p.322-327, 1999.

SILVA, N.; ABUSLEME, L.; BRAVO, D. et al. Host response mechanisms in periodontal diseases. J. Appl. Oral Sci., v.23, n.3, p.329-355, 2015. doi: 10.1590/1678-775720140259.

SOCRANSKY, S.S.; HAFFAJEE, A.D.; CUGINI, M.A. et al. Microbial complexes in subgingival plaque. J. Clin. Periodontol., v.25, n.2, p.134-144, 1998. doi: 10.1111/j.1600-051x.1998. tb02419.x.

TURNER, C. et al. Oral health status of Russian children with unilateral cleft lip and palate. Cleft Palate Craniofac J., v.35, n.6, p.489-494, 1998. doi: 10.1597/1545-1569_1998_035_0489 ohsore 2.3.co_2.

VALM, A.M. The structure of dental plaque microbial communities in the transition from health to dental caries and periodontal disease. J. Mol. Biol., v.431, n.16, p.2957-2969, 2019. doi: 10.1016/j.jmb.2019.05.016.

VEIGA, K.A. et al. Caries experience and periodontal status in children and adolescents with cleft lip and palate. Pediatr. Dent., v.39, n.2, p.139-144, 2017. 